\title{
Nitric Oxide/cGMP-Mediated Protein Kinase A Activation in the Antennal Lobes Plays an Important Role in Appetitive Reflex Habituation in the Honeybee
}

\author{
Uli Müller ${ }^{1}$ and Herbert Hildebrandt ${ }^{2}$ \\ 1/nstitut für Biologie der Freien Universität Berlin, Neurobiologie, 14195 Berlin, Germany, and 2Institut für Zoologie der \\ Universität Stuttgart-Hohenheim, 70593 Stuttgart, Germany
}

Habituation, a form of non-associative learning, is observed throughout the animal kingdom. However, in contrast to associative learning, little is known about the underlying molecular mechanisms. Using the appetitive proboscis extension reflex in honeybees, we show that the cAMP-dependent protein kinase $A(P K A)$ in the antennal lobe (AL) is implicated in the graded decline of behavioral response during habituation. Repeated stimulation leads to a slow and gradual increase in PKA activity superimposed on a fast transient PKA activation induced by each stimulus. These temporally distinct components of PKA activation are pharmacologically dissectible and are restricted to the $\mathrm{AL}$ on the stimulated side. Whereas the transient PKA activation induced by each stimulus requires monoaminergic transmission, the slow component of PKA activation is medi- ated by the nitric oxide (NO)/cGMP system. Local manipulation of the slow component of PKA activation in single ALs specifically interferes with the dynamic of habituation on the corresponding side. Our results provide strong evidence that NO/ cGMP-mediated PKA activation in each AL contributes to temporal signal integration during habituation. Dishabituation by a sensory stimulus or spontaneous recovery from habituation does not require the PKA cascade. This provides evidence that the mechanisms underlying dishabituation and spontaneous recovery differ from those underlying temporal signal integration during habituation of the proboscis extension response.

Key words: antennal lobes; cAMP-dependent protein kinase; dishabituation; habituation; insect; plasticity; recovery from habituation; second messenger
Habituation is a gradual decline in a behavioral response resulting from repeated stimulation of a reflex pathway (Thompson and Spencer, 1966; Groves and Thompson, 1970). Paradoxically, compared with more complex forms of learning, the molecular mechanisms underlying habituation are not well understood. Most of what we know about the neural basis of habituation comes from investigations of the gill and siphon withdrawal reflex in Aplysia (Castellucci et al., 1970; Carew et al., 1972) and the crayfish escape reflex (Krasne, 1969).

Early work in Aplysia demonstrated that repeated electrical stimulation of isolated sensory neurons causes a decrease in the calcium current and thus a reduction of transmitter release (Boyle et al., 1982; Byrne, 1982; Rayport and Schacher, 1986). This was in agreement with the observation that habituated animals show fewer vesicles in the active zone of sensory neurons than control animals do (Bailey and Chen, 1983, 1988) and pointed to a critical role of homosynaptic depression in sensory neurons. However, recent work in Aplysia show that sensory neurons exhibit heterosynaptic facilitation rather than homosynaptic depression during habituation (Stopfer and Carew, 1996). This provides evidence that plastic changes contributing to habituation occur at the level of interneurons and suggests that several mechanisms distributed within a neural circuit contribute to habituation.

Received April 12, 2002; revised June 26, 2002; accepted July 12, 2002.

This work was supported by Deutsche Forschungsgemeinschaft Sonderforschungsbereich 515/C3. We thank R. Menzel for helpful suggestions on this manuscript and M. Wurm for help with this manuscript.

Correspondence should be addressed to Uli Müller, Freie Universität Berlin, Institut für Biologie, Neurobiologie, Königin-Luise-Strasse 28/30, 14195 Berlin, Germany. E-mail: muelleru@zedat.fu-berlin.de.

Copyright (C) 2002 Society for Neuroscience $0270-6474 / 02 / 228739-09 \$ 15.00 / 0$
The neural circuit implicated in habituation of mechanosensory signals has been identified in great detail in the nematode Caenorhabditis elegans (Chalfie et al., 1985; Wicks and Rankin, 1995; Rose and Rankin, 2001). Both the touch withdrawal response and the tap withdrawal response use a very similar network of identified neurons. Although the connectivity and the roles of each of these identified neurons are characterized, the molecular mechanisms contributing to habituation of the withdrawal response are unknown.

In Drosophila, a variety of different habituation paradigms, including proboscis extension reflex (PER) (Duerr and Quinn, 1982), landing response (Asztalos et al., 1993), visual escape jump (Engel and Wu, 1996, 1998), and a reflex controlling leg position (Jin et al., 1998) have been investigated. The neural circuits that mediate the behaviors are very complex and hardly accessible using physiological techniques. These complex circuits, and the fact that all mutants with defects in signaling pathways implicated in synaptic plasticity and learning (Davis, 1996) also affect habituation, make it difficult to dissect the specific contribution of the various signaling cascades with respect to habituation.

In this work, we demonstrate that the cAMP cascade contributes to habituation of the PER in honeybees (Braun and Bicker, 1992). Whereas a single appetitive chemosensory stimulation applied to one antenna elicits the extension of the proboscis, repeated stimulations lead to a gradual decrease in responsiveness. We now show that the decrease in response probability during habituation correlates with a slow and gradual increase in protein kinase A (PKA) activation in the stimulated antennal lobe (AL). The latter is mediated by the nitric oxide (NO)/cGMP system within the circuitry of each AL. Interestingly, the PKA cascade in the AL selectively contributes to temporal signal 
integration during habituation but is not involved in mechanisms underlying dishabituation and recovery from habituation.

\section{MATERIALS AND METHODS}

Materials. ODQ [1H-(1,2,4)oxadiazolo(4,3-a)quinoxalin-1-one] was purchased from Alexis (Grünberg, Germany). KT5720, KT5823, KN 93, bisindoylmaleinimide, Rp-8-BrcAMPS, Rp-8-BrcGMPS, caged cAMP, and caged cGMP were from Calbiochem (Bad Soden, Germany). Caged nitric oxide I was from Molecular Probes Europe (Leiden, The Netherlands). $\left[\gamma_{-}{ }^{32} \mathrm{P}\right] \mathrm{ATP}(5000 \mathrm{Ci} / \mathrm{mmol})$ was purchased from NEN Life Science Products (Brussels, Belgium). PKA inhibitor peptide, PKI (622), N-nitro-L-arginine (NOArg), and all other chemicals were obtained from Sigma (Deisenhofen, Germany).

Animal treatment and sensory stimulation. Adult foraging honeybees (Apis mellifera carnica) were caught the day before the experiment, immobilized by cooling, and harnessed in metal tubes with a strip of tape between the head and the thorax. In the evening, they were fed with sucrose solution $(1.4 \mathrm{M})$ to satiation and kept in darkness in a container at $70 \%$ relative humidity and $20-25^{\circ} \mathrm{C}$. Three hours before the experiment, they were fed with $3 \mu$ l of sucrose solution $(1.4 \mathrm{M})$. For sensory stimulation, sucrose solution $(0.6 \mathrm{M})$ was applied to one antenna using a wooden toothpick. Stimulus duration was $\sim 0.3-0.5 \mathrm{sec}$. Unless otherwise indicated, an interstimulus interval (ISI) of $2 \mathrm{sec}$ was used. Habituation is defined using a criterion of five consecutive response failures. To determine PKA activity, bees were frozen in liquid nitrogen at times as indicated in Results. To test for differences between the different groups, ANOVAs were performed followed by appropriate post hoc tests.

Injection procedures. Systemic injections into the hemolymph $(1 \mu \mathrm{l})$ and local injections into the AL $(1 \mathrm{nl})$ were performed as described previously (Müller and Hildebrandt, 1995). For injections into the AL, glass microcapillaries connected to a Picosprizer (General Valve, Fairfield, NJ) were used. The microcapillary was introduced through a small window cut dorsally just above the antennae in the bee's head capsule. Coinjection of bromophenol blue enabled the visual control of AL injections through the window in the cuticula using a stereo microscope. Only animals that show a distinct staining restricted to the injected AL ( $>70 \%$ of the injected animals) were used for the experiment. After 5 min recovery, response to single stimuli was tested. Animals showing no response were discarded $(<5 \%)$.

Photolyzing substances in the antennal lobes in vivo. To allow illumination of the AL, a small window was cut into the head capsule dorsally above the antennae $1 \mathrm{~d}$ before the experiment. Tracheae and neurolemma covering the brain were left intact. Ringer's solution alone or Ringer's solution containing the caged compound was injected into the hemolymph $20 \mathrm{~min}$ before photolyzing. A UV flash (T.I.L.L. Photonics, Planegg, Germany) was used as light source. The collimated output of the UV flash enters the photoadapter port of a binocular $(40 \times)$ used to focus the flash on an AL. An aperture with a hole in the plane of focus enables local illumination of a single AL immediately before the habituation session. The intensity of illumination and thus the appropriate amount of substance released in the AL was determined by measuring the effect on PKA activation in the appropriate AL (Müller, 2000).

Preparation of antennal lobes. Heads frozen in liquid nitrogen were lyophilized, and the ALs were dissected under continuous liquid nitrogen cooling (Hildebrandt and Müller, 1995a). Each AL was transferred into a glass capillary containing $10 \mu \mathrm{l}$ of extraction buffer $(50 \mathrm{~mm}$ Tris-HCl, pH 7.5, $0.1 \mathrm{~m} \mathrm{NaCl}, 1 \mathrm{~mm}$ EDTA, $1 \mathrm{~mm}$ EGTA, and $10 \mathrm{~mm}$ 2-mercaptoethanol) frozen in liquid nitrogen. The tissue was homogenized on the surface of the frozen buffer, and the capillaries were stored in liquid nitrogen until subsequent phosphorylation.

Determination of $P K A$ activity. The PKA activity in the AL was determined as described previously (Müller, 2000). The tissue in the capillaries was thawed and immediately plunged into $10 \mu \mathrm{l}$ of phosphorylation buffer containing $1 \mu \mathrm{Ci}$ of $\left[\gamma^{32} \mathrm{P}\right] \mathrm{ATP}(5000 \mathrm{Ci} / \mathrm{mmol}), 30 \mu \mathrm{M}$ ATP, $20 \mathrm{mM} \mathrm{MgCl}$, $1 \mathrm{mM}$ EGTA, and $10 \mathrm{~mm}$ mercaptoethanol in $50 \mathrm{~mm}$ Tris-HCl, pH 7.5, and $1 \mu \mathrm{g}$ of the PKA-specific substrate protein I-1. After $20 \mathrm{sec}$ at $20^{\circ} \mathrm{C}$, reactions were terminated by adding $5 \mu \mathrm{l}$ of SDS-PAGE loading buffer (0.5 M Tris-HCl, pH 6.8, with 5\% mercaptoethanol, $5 \%$ SDS, $20 \%$ glycerol, and $0.1 \%$ bromophenol blue). After SDS-PAGE, ${ }^{32} \mathrm{P}$ incorporation into protein $\mathrm{I}-1$ was quantified as described previously (Hildebrandt and Müller, 1995a,b; Müller, 2000).

\section{RESULTS}

\section{The cAMP/PKA cascade in the antennal lobes contributes to the habituation of the proboscis extension reflex}

To identify the signaling cascade that mainly contributes to the habituation of the PER, we blocked different protein kinases known to play a role in neural plasticity. The inhibitors were tested for their specificity by appropriate kinase assays in vitro. After dilution within the animals, the concentrations used for injections inhibit the respective kinase activity by $>80 \%$ as determined in the in vitro assays.

Only inhibition of the cAMP-dependent protein kinase (PKA) by inhibitors such as KT5720 (Fig. $1 A$ ) or Rp-BrcAMPS (Fig. $1 B$ ) has an effect on habituation. As expected, activation of PKA by systemic injection of BrcAMP has the opposite effect and accelerates habituation (Fig. 1B). Blocking cGMP-dependent protein kinase (PKG) with KT5823, $\mathrm{Ca}^{2+} /$ phospholipiddependent protein kinase $\mathrm{C}$ (PKC) with bisindolylmaleinimide, or $\mathrm{Ca}^{2+} /$ calmodulin-dependent protein kinase II (CaMKII) with KN 93, does not interfere with habituation. Neither excitability of the PER nor dishabituation is affected by the kinase inhibitors used.

Evidence from recent work suggests that the main circuit mediating habituation of the PER is located in the ALs (Braun and Bicker, 1992; Müller and Hildebrandt, 1995). Therefore, we tested whether local manipulation of PKA activity in the ALs causes the same effect as the systemic application of drugs. Injection of Rp-BrcAMPS, an inhibitor of PKA, into one AL affects habituation at the ipsilateral side, whereas habituation of the contralateral side remains normal (Fig. 1B). Accordingly, local uncaging of cAMP in one AL accelerates the habituation only at the ipsilateral side (Fig. 1B). In both cases, the observed effects are not distinguishable from those caused by systemic injection. This points to a major contribution of the cAMP/PKA cascade in the ALs to habituation processes.

\section{Repeated sucrose stimulation to an antenna induces a gradual increase in PKA activity in the corresponding antennal lobe}

We demonstrated previously that a single sucrose stimulus applied to an antenna of a naive animal causes a very rapid and transient increase in PKA activity in the AL on the stimulated side (Hildebrandt and Müller, 1995a,b). Mechanosensory or odor stimulation does not affect PKA activity in the ALs. Because interference with the cAMP/PKA cascade affects the time course of habituation (Fig. 1B) but not the response to the initial stimulus, we measured the temporal dynamics of PKA activation in the AL during the habituation session (Fig. $2 A$ ).

The temporal dynamics of PKA activation induced by the first and the fifth stimulus is similar (Fig. 2B): in both cases, there is a fast transient increase in PKA activity that reverts within $1.5 \mathrm{sec}$ back to the basal level of nonstimulated control animals (dashed line). Application of 30 stimuli in succession (ISI, $2 \mathrm{sec}$ ), which leads to a drastic reduction in response probability (Fig. $2 A$ ), drastically changes the temporal dynamics of PKA activation. In addition to the quickly decaying PKA activity induced by each stimulus, an elevated PKA activity is detectable (Fig. $2 B$ ). Because the latter decays over minutes (see also Fig. 4), we define it as slowly decaying component of PKA activation. In agreement with the behavioral results, repeated sucrose stimulation affects only PKA activation in the AL ipsilateral to the stimulated antennae. 

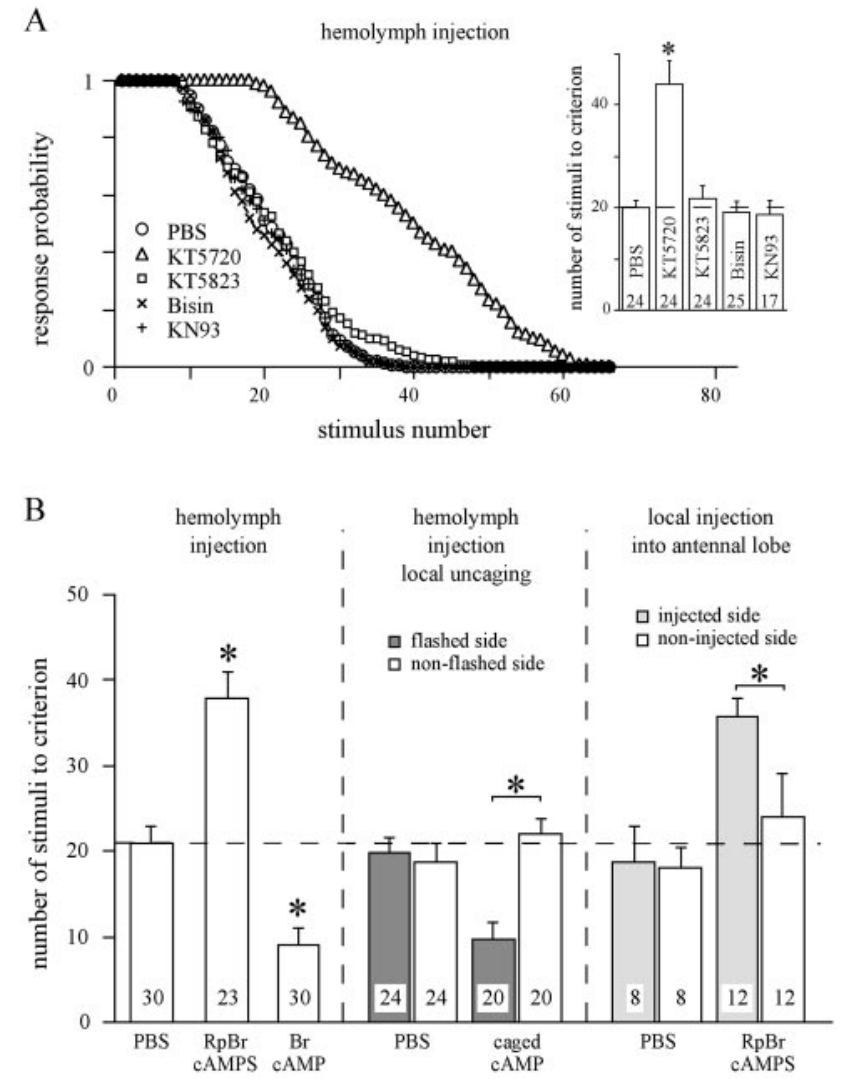

Figure 1. Involvement of second-messenger systems in habituation of the proboscis extension response. $A$, Thirty minutes before the habituation session, animals were injected (1 $\mu$ l each) with PBS alone or PBS containing the PKA inhibitor KT5720 (100 $\mu \mathrm{M})$, the PKG inhibitor KT5823 $(500 \mu \mathrm{M})$, the PKC inhibitor bisindoylmaleinimide $(1 \mathrm{mM})$, or the CaMKII inhibitor KN93 (1 mM). For habituation, one antenna was repeatedly stimulated (ISI, $2 \mathrm{sec})$ with sucrose solution $(0.6 \mathrm{M})$. The data points for each stimulus represent the average of the response probability of all animals in the respective group. The inset shows the mean \pm SEM of the number of stimuli required to fulfill the habituation criterion. Number of animals is indicated in each bar (ANOVA, $p<0.0001 ;{ }^{*} p<$ 0.0001 , Scheffe's post hoc test). $B$, In the first experiment, animals received hemolymph injections (1 $\mu \mathrm{l}$ each) of PBS, Rp-BrcAMPS $(500 \mu \mathrm{M})$, or BrcAMP $(500 \mu \mathrm{M})$. After $20 \mathrm{~min}$, the animals were habituated (ANOVA, $p<0.0001$; Scheffe's post hoc test, ${ }^{*} p<0.0001$, Rp-BrcAMPS- and BrcAMP-injected groups differ significantly from each other and from the PBS-injected group). In a second experiment, the animals were injected with either $(1 \mu \mathrm{l}$ each) PBS or caged cAMP $(500 \mu \mathrm{M})$. After $20 \mathrm{~min}$, animals received light flashes onto one AL. Twenty seconds later, habituation was tested on both sides of each animal. In the third experiment, groups of animals receive local injections $(2 \mathrm{nl})$ of either PBS or RpBrcAMPS $(100 \mu \mathrm{M})$ into one AL. Five minutes after AL injection, the animals were habituated on either side [ANOVA reveals significant differences between the flashed or injected side $(p<0.0001)$ but not the nonflashed or non-injected sides $(p=0.34)$, respectively $\left({ }^{*} p<0.0001\right.$, paired $t$ test)].

Based on the finding that a single sucrose stimulus induces an octopamine-mediated, transient PKA activation in the AL (Hildebrandt and Müller, 1995a), we tested how depletion of monoamines interferes with habituation of PER and the dynamics of PKA activation during a habituation session. Although reserpine injections ( $2 \mu \mathrm{g}$ /animal, $18 \mathrm{hr}$ before test) slow down the spontaneity and the movement of the proboscis, sucrose stimulation fails to induce a PER in only a few animals $(<7 \%)$. These animals were excluded from the experiment. The considerably higher ratio of nonresponding animals $(\sim 30 \%)$ after re- serpine injection observed by Braun and Bicker (1992) is most likely attributable to the different interval between injection and test (12 hr in contrast to $18 \mathrm{hr}$ in our study).

In agreement with our previous findings, monoamine depletion eliminates the fast component of PKA activation induced by each sucrose stimulus (Fig. 2B). Interestingly, the slowly decaying component of PKA activation induced by repeated stimulation (Fig. $2 B$ ) and habituation of PER (Fig. $2 A$ ) is unaffected. Dishabituation and responsiveness to sucrose stimuli do not differ between the group treated with reserpine and the group injected with the vehicle (DMSO) alone. Moreover, injection of the vehicle (DMSO) affects neither basal PKA activity nor the fast transient PKA activation after sucrose stimulation (data not shown). Thus, the quickly and the slowly decaying components of PKA activation are pharmacologically dissectible. Whereas the quickly decaying PKA activation induced by each stimulus requires monoamine transmission, the slowly decaying PKA activation induced only by repeated stimulation is independent of monoamine-mediated processes.

\section{Response probability during habituation correlates with the slowly decaying PKA activity}

To support the idea that changes in response probability during habituation correlate with the slowly decaying component of PKA activity, response probability and PKA activation in the AL were determined. The animals were subjected to two subsequent habituation sessions separated by a dishabituating stimulus (Fig. 3). The PKA activity in the ALs during the time course of habituation was determined in identically treated groups of animals killed at the end of the stimulus number indicated on the abscissa. To selectively determine the slow component of PKA activity, the animals were shock frozen in liquid nitrogen $5 \mathrm{sec}$ after the end of the indicated stimulus number. During the time course of the first habituation session, the response probability decreases from 1.0 to 0.08 within 30 stimuli (Fig. $3 A$ ). The decrease in response probability correlates with a gradual increase of the slowly decaying PKA activity in the AL of the stimulated side (Fig. 3B). PKA activity in the AL of the unstimulated side does not change and remains at the level of unstimulated control animals.

A dishabituating stimulus to the contralateral antenna resets both the response probability and the increased level of the PKA activity in the AL of the formerly stimulated side. The second habituation session, immediately applied to the previously habituated side, shows a faster decrease in response probability (Fig. $3 A$ ) and a faster increase in the levels of the slow component of PKA activity (Fig. 3B). For both habituation sessions, the response probability is highly correlated with the level of the slowly decaying component of PKA activity (Fig. $3 C$ ). This is also true for ISIs of 1 and $3 \mathrm{sec}$ (data not shown).

\section{Spontaneous recovery from habituation}

Another characteristic feature of habituation is the spontaneous recovery of the behavioral response. To test whether the slow component of PKA activity in the AL also correlates with the recovery at the behavioral level, both parameters were measured. Animals were habituated until they reach the criterion and, after distinct times of recovery, were habituated once more. Immediately after the first habituation session, the response probability of the stimulated side is very low (Fig. 4A). Approximately 5-6 min after the initial habituation session, recovery is complete and the number of stimuli required for the second habituation is similar 
Figure 2. Habituation of the proboscis extension response and the dynamic of PKA activation in the antennal lobes. $A$, Animals were injected with $1 \mu \mathrm{l}$ of PBS, DMSO, and DMSO containing $2 \mu \mathrm{g}$ of reserpine $18 \mathrm{hr}$ before habituation. Sucrose stimuli were applied to one antenna until the habituation criterion was fulfilled. The data points for each stimulus represent the average of the response probability of all animals in the respective group (20 animals each). Habituation kinetics did not differ between the groups (ANOVA, $p=0.95$ ). $B$, PKA activity in the antennal lobes of the stimulated and the unstimulated sides was determined at different times after the 1st, 5th, and 30th stimulus (see arrows in $A$ ). In each experiment, values were normalized to PKA activity in unstimulated animals, which are defined as the relative PKA activity of 1 (dashed lines). Each data point represents the mean \pm SEM of relative PKA activity of at least eight independent measurements [1st and 5th stimulus: ANOVA, $p<0.0001 ; 0.5$ sec after stimulation, the PBS-injected group significantly differs from the corresponding unstimulated $\mathrm{AL}\left({ }^{*} p<0.001\right.$, paired $t$ test $)$ and all other means $(p<0.003$, unpaired $t$ test); 30th stimulus: ANOVA, $p<0.0001$; at

each time point, the means of the stimulated ALs (PBS- and reserpine-injected groups) significantly differ from their respective unstimulated ALs $\left({ }^{*} p<0.001\right.$, paired $t$ test)]. Value at $0.5 \mathrm{sec}$ (PBS-injected animals) significantly differs from all other values $(p<0.003$, unpaired $t$ test $)$

to that of the first session. The half-time for recovery is $\sim 2 \mathrm{~min}$ and does not differ between subgroups of animals that reach the criterion in the first habituation session with a mean of 12 stimuli $\left(t_{(0.5)}=2 \mathrm{~min} ; n=18\right)$ or with a mean of $28 \operatorname{stimuli}\left(t_{(0.5)}=1.9\right.$ $\min ; n=15)$.

The slow component of PKA activity in both ALs was measured at distinct recovery times in animals treated in parallel (Fig. $4 B$ ). Immediately after the first habituation session, the slow component of PKA activity in the AL of the previously habituated side is at its maximum. With recovery of response probability over time, the level of PKA activity decays until it reaches the level of the nonhabituated control or that of the unstimulated control (Fig. $4 B$ ). As shown in Figure $4 C$, the response probability and the level of the slow component of PKA activity in the AL are highly correlated (Fig. 4C).

\section{The NO/cGMP system mediates gradual PKA activation during habituation}

Given the strong correlation between the response probability in habituation and the PKA activity in the AL, drugs interfering with the cAMP/PKA pathway were tested for their effects on habituation. Systemic injection of membrane-permeable BrcAMP significantly accelerates habituation, whereas injection of Rp-BrcAMPS, a competitive antagonist of cAMP, slows down habituation compared with PBS-injected animals (Fig. 5A). The same result was obtained using BrcGMP and Rp-BrcGMPS, which preferentially interfere with cGMP-dependent protein kinase PKG but also have effects on PKA (Müller, 2000) (Fig. 5B). An implication of PKG in habituation is very unlikely, because only blocking of PKA activity (KT5720) but not inhibition of PKG activity (KT5823) causes a slowdown in habituation (Fig. $5 C$ ). This suggests that BrcGMP and Rp-BrcGMPS most likely act on PKA, although a role of nucleotide-gated channels cannot be excluded.

In agreement with our previous findings (Müller and Hildebrandt, 1995), inhibition of NO synthase (NOS) causes a slow- down in habituation (Fig. 5D). The function of NO in habituation is supported by the observation that scavengers of NO slowdown response probability during habituation with the same kinetic as the other drugs interfering with habituation (Fig. 5E). Because blocking the NO-regulated soluble guanylate cyclase using ODQ causes the same effect on habituation, the physiological function of NO is most likely mediated via cGMP (Fig. 5D). This is in agreement with our finding that BrcGMP and Rp-BrcGMPS cause the same effect as BrcAMP and Rp-BrcAMPS (Fig. 5, compare $A, B)$.

The high correlation between the slow component of PKA activation in the AL and the response probability (Fig. 5, right panels) suggest that repeated stimulation modulates PKA activity in the AL via the NO/cGMP system. Again, none of the drugs used affect response probability to the initial sucrose stimuli or dishabituation.

\section{The NO/cGMP system in the circuitry of each antennal lobe separately contributes to the gradual changes in response probability and PKA activation during habituation}

In the previous experiments, the drugs interfering with the $\mathrm{NO} /$ cGMP system were injected into the hemolymph and can thus exert their effects in brain areas other than the AL. To prove that the action of NO and cGMP is restricted to the circuit of a single AL, we locally uncaged cGMP and NO in a single AL shortly before the habituation session and measured the effects on response probability. Both the uncaging of NO and of cGMP in a single AL cause a faster decrease in response probability on the side ipsilateral to the stimulated antenna (Fig. 6). Habituation on the contralateral side to the treatment does not differ from PBSinjected animals. This clearly demonstrates that the NO/cGMP system in each AL separately is implicated in the modulation of habituation on the corresponding side. To address the question whether NO acts via the guanylate cyclase, we systemically blocked the guanylate cyclase and locally uncaged NO or cGMP 
A
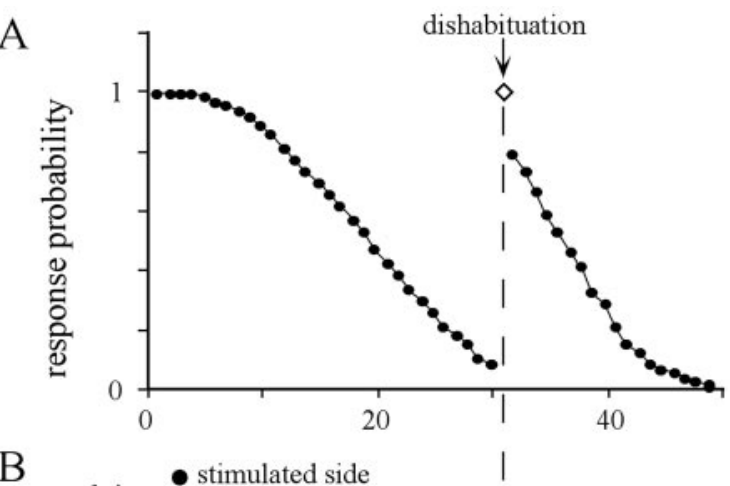

$\mathrm{B}$

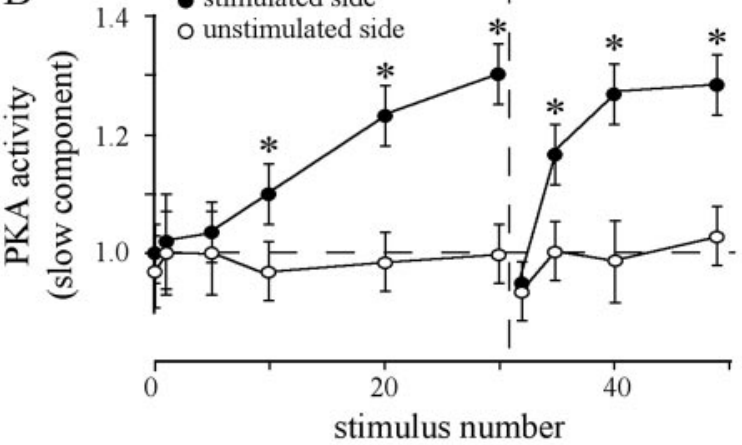

$\mathrm{C}$

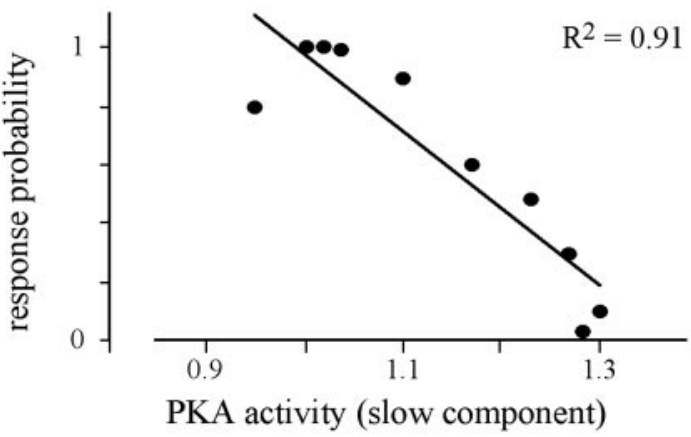

Figure 3. Dishabituation by an appetitive stimulus and PKA activity in the AL. $A$, Animals received 30 sucrose stimuli (ISI, $2 \mathrm{sec}$ ) to one antenna, followed by a dishabituating stimuli applied to the contralateral antenna (arrow), and a second session of repetitive sucrose stimulations to the previously stimulated antenna. The values represent the average of the response probability of 15 animals. $B$, The slow component of PKA activity in both antennal lobes was determined $5 \mathrm{sec}$ after the stimulus number indicated. Values were normalized to PKA activity in unstimulated animals, which are defined as the relative PKA activity of 1 (dashed lines). Each data point represents the mean \pm SEM of the PKA activity of at least seven measurements (ANOVA: stimulated side, $p<0.0001$; unstimulated side, $p=0.88 ;{ }^{*} p<0.008$, paired $t$ test, stimulated vs unstimulated side). $C$, The slow component of PKA activity determined in the stimulated AL (data from $B$ ) is highly correlated with the corresponding response probability (data from $A$ ).

in a single AL before habituation. Only local uncaging of cGMP, but not the release of NO in the AL, rescues the effect of systemically inhibited soluble guanylate cyclase on habituation (Fig. 6). This strongly suggest that NO in the AL acts exclusively via the soluble guanylate cyclase.

None of the treatments affect response probability to a single sucrose stimulus or dishabituation. This and the previous results provide strong evidence that NO/cGMP-mediated PKA activation in the circuitry of each AL contributes to changes in response decrement but is itself not involved in mechanisms of reflex release and dishabituation.
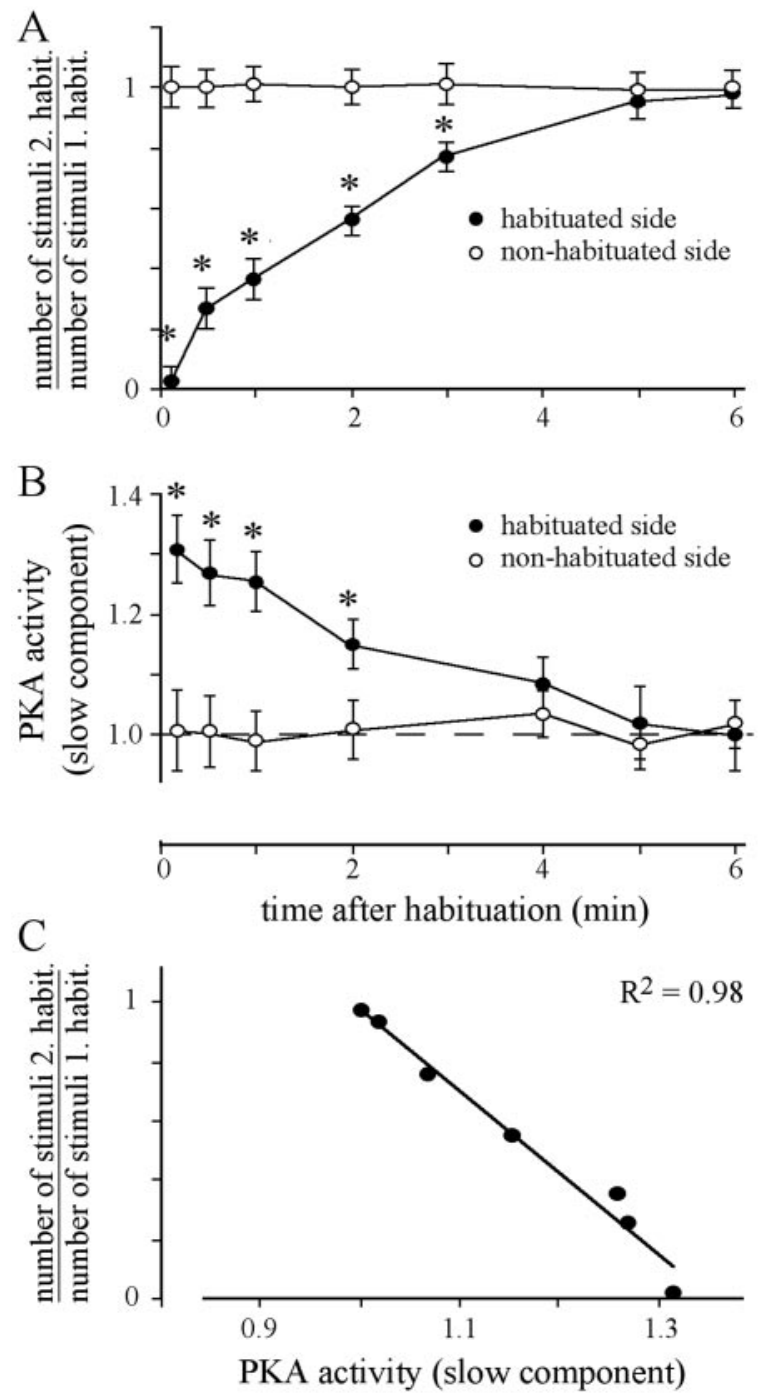

Figure 4. Spontaneous recovery from habituation and slow component of PKA activity. $A$, Animals were habituated until the criterion of five failures was met. After recovery for the indicated times, the animals received a second habituated session. In the second session, either the previously habituated side or the previously nonhabituated side was stimulated. For each animal, the number of stimuli required for the second habituation session was divided by the number of stimuli necessary in the first habituation session. Each data point represents the mean \pm SEM of at least 18 animals (ANOVA: habituated side, $p<0.0001$; nonhabituated side, $p=0.99$; ${ }^{*} p<0.006$, paired $t$ test, habituated vs nonhabituated side). $B$, After habituation and recovery for the indicated times, the slow component of PKA activity was measured in each AL. The values were normalized with respect to PKA activity in unstimulated animals handled identically (dashed lines). Each data point represents the mean \pm SEM of at least 10 measurements (ANOVA: habituated side, $p<$ 0.0005 ; nonhabituated side, $p=0.97 ;{ }^{*} p<0.003$, paired $t$ test, habituated vs nonhabituated side). $C$, Correlation between the slow component of PKA activity in the AL of the habituated side and the respective relative response probability of the habituated side for the tested recovery times.

\section{Spontaneous recovery from habituation does not depend on the PKA system}

In a final point, we addressed the question whether the PKA system contributes to mechanisms of spontaneous recovery from habituation. Several reports demonstrate that spontaneous recovery depends on the ISI used for habituation (for review, see Rankin, 1994; Rose and Rankin, 2001). Shorter ISIs that lead to 

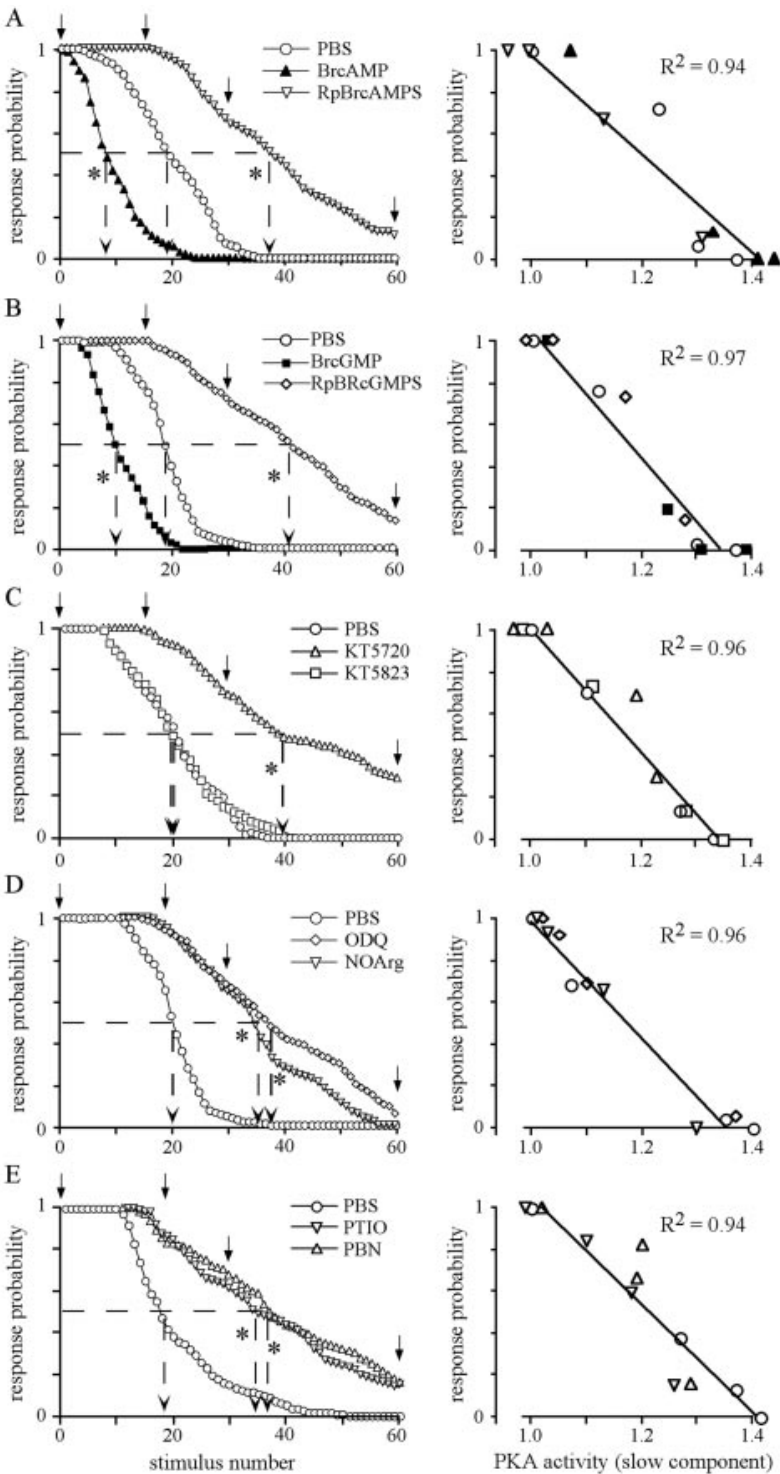

Figure 5. The slow component of PKA activation during habituation is mediated by the NO/cGMP pathway. Thirty minutes before habituation, the animals receive systemic injections $(1 \mu \mathrm{l})$ of the following solutions: $A$, BrcAMP (500 $\mu \mathrm{M})$ and Rp-BrcAMPS (500 $\mu \mathrm{M})$; B, BrcGMP (500 $\mu \mathrm{M})$ and Rp-BrcAMPS $(500 \mu \mathrm{M}) ; C$, inhibitors of PKA (KT5720, $100 \mu \mathrm{M})$ or PKG (KT5823, $500 \mu \mathrm{M}) ; D$, inhibitors of NO synthase (NOArg, $500 \mu \mathrm{M}$ ) or soluble guanylate cyclase (ODQ, $500 \mu \mathrm{M})$; and $E$, the NO scavengers 2-phenyl-4,4,5,5-tetramethylimidazoline-1-oxyl-3-oxide (1 $\mathrm{mm})$ and $N$-tert-butyl- $\alpha$-phenylnitrone (1 mM) (Alexis, Grünberg, Germany). In all cases, animals injected with $1 \mu \mathrm{l}$ of PBS were used as controls. The data points for each stimulus represent the average of the response probability of all animals in the respective group $(n>24)$. The mean of the number of stimuli required for habituation is indicated by arrows pointing to the abscissa (dashed lines). (ANOVA, $p<0.0001$; Scheffe's post hoc test, $p<$ 0.0001 ; ${ }^{*}$ indicates groups that significantly differ from PBS-injected groups). The slow component of the PKA activity in the AL of the stimulated side was determined after the 1st, 15th (or 20th in $D$ and $E$ ), 30 th, and 60 th stimulus (marked by arrows). As shown in the right column, the means of the slow PKA activity in the AL of the stimulated side $(n \geq$ 5 for each time point and treatment) highly correlate with the corresponding response probability.

more rapid habituation than longer ISIs also lead to a faster recovery from habituation. As demonstrated previously (Braun and Bicker, 1992; Müller and Hildebrandt, 1995) and in agreement with other habituation paradigms, short ISIs $(1 \mathrm{sec})$ lead to

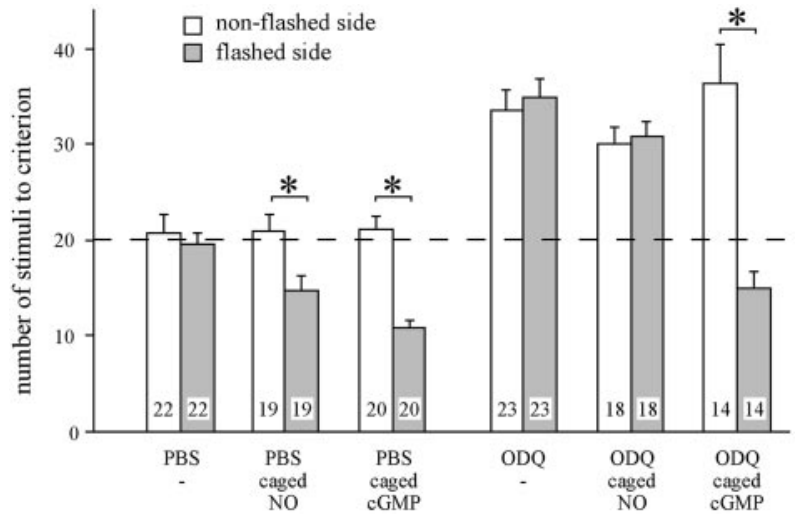

Figure 6. Dynamics of habituation is modulated by the NO/cGMP system in the circuitry of a single AL. Fifteen minutes before habituation, groups of animals were injected ( $1 \mu$ l into the hemolymph) with PBS, caged NO $(500 \mu \mathrm{M})$, and caged cGMP $(500 \mu \mathrm{M})$, or with ODQ $(500 \mu \mathrm{M})$, ODQ plus caged NO, and ODQ plus caged cGMP. For local NO or cGMP release, animals receive light flashes focused onto one AL followed by habituation of the flashed and the nonflashed side. The values represent the mean \pm SEM of the number of stimuli needed to meet the habituation criterion for each side. Number of animals tested is indicated in the bars [nonflashed side: ANOVA, $p<0.0001$; Scheffe's post hoc test, $p<0.004$; groups injected with ODQ, ODQ plus caged NO, and ODQ plus caged cGMP differ from groups injected with PBS, caged NO, and caged cGMP; flashed side: ANOVA, $p<0.0001$; ${ }^{*}$ indicates significant differences between habituated and nonhabituated side $(p<0.002$, paired $t$ test)].

a more rapid habituation than longer ISIs $(3 \mathrm{sec}$ ) (Fig. 7A, 1st habituation session). Independent of the ISIs, injection of BrcAMP causes the same relative acceleration of habituation (Fig. 7A) (mean stimuli number of first habituation session BrcAMP/mean stimuli number of first habituation session PBS: $1 \mathrm{sec}$ ISI, 7,9/12 $=0.66 ; 2 \mathrm{sec}$ ISI, $13 / 20=0.65 ; 3 \mathrm{sec}$ ISI, $22 / 36=0.61)$.

In contrast to findings in other habituation paradigms, recovery from habituation does not depend on the ISIs, at least in the case of the ISIs used in this study. In all cases, the second habituation session after a recovery period of 2 min (half-time as determined in Fig. 4) requires approximately half the trials of the previous first habituation session (Fig. $7 B$ ). Because this also applies to BrcAMP-injected animals, the cAMP/PKA system seems not to contribute to mechanisms underlying spontaneous recovery from habituation.

\section{DISCUSSION}

Although cAMP-dependent mechanisms seem to play a conserved role in processes leading to long-lasting neuronal changes and long-term memory (LTM) (Schacher et al., 1988; Abel et al., 1997; Müller, 2000), the function of the cAMP cascade in mechanisms underlying non-associative learning is controversial and not well documented. We show here that, in addition to its function in the induction of long-term associative memory (Müller, 2000), the cAMP cascade in the honeybee is implicated in very distinct aspects of response habituation. In the circuitry of each antennal lobe, the cAMP/PKA system is selectively implicated in processing repeated appetitive stimulation during response habituation but does not contribute to dishabituation or spontaneous recovery from habituation.

The role of second-messenger cascades in habituation Work on Drosophila mutants with defects in the cAMP cascade points to a role of the cAMP cascade in habituation (Duerr and 

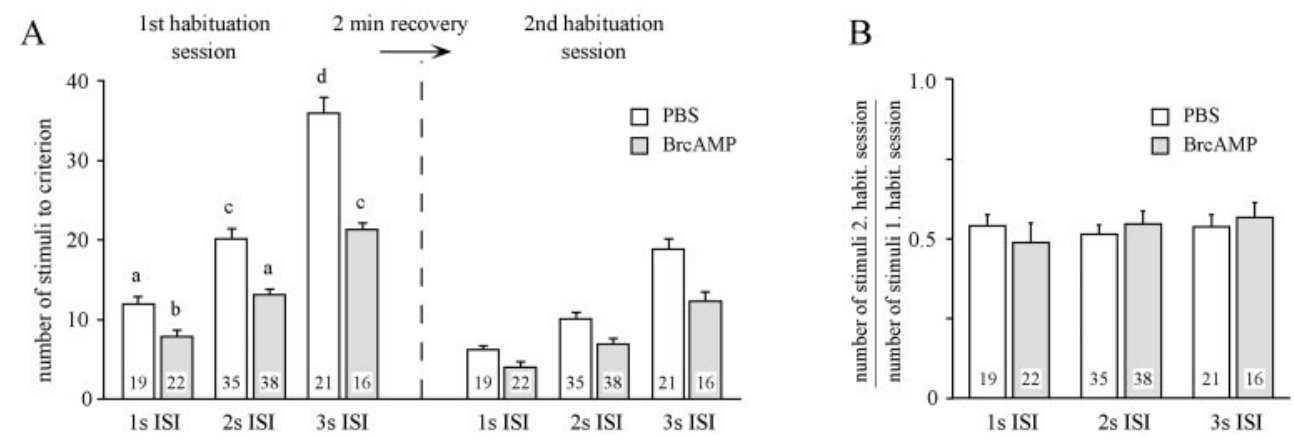

Figure 7. Spontaneous recovery from habituation does not depend on PKA activity. $A$, Groups of animals were injected ( $1 \mu$ linto the hemolymph) $15 \mathrm{~min}$ before habituation with either PBS or PBS containing $500 \mu \mathrm{M}$ BrcAMP. Using the indicated ISI, different groups of animals were habituated until the criterion of five failures was met. After 2 min recovery, the same animals receive a second habituation session by stimulating the same antenna. The values present the mean \pm SEM of stimuli necessary to attain the criterion in the first habituation session (left) and the second habituation session $($ right $)$. The number of animals is indicated in the bars. First habituation session: ANOVA, $p<0.0001$; values indicated with different letters significantly differ from each other $(p<0.001$ unpaired $t$ test). Means of the first habituation session significantly differ from that of the corresponding second habituation session (ANOVA with repeated measures: $p<0.0001 ; p<0.0001$, paired $t$ test). $B$, For each animal, the number of stimuli required for habituation in the second habituation session is proportional to the number of stimuli necessary for the first habituation session. (ANOVA, $p=0.83$ ).

Quinn, 1982; Rees and Spatz, 1989; Engel and Wu, 1996). However, it is difficult to extract from these findings a consistent picture of how the cAMP cascade contributes to mechanisms underlying habituation. For example, although the effects of dunce (a phosphodiesterase that reduces cAMP levels; Byers et al., 1981) and rutabaga (an adenylyl cyclase that elevates cAMP levels; Livingstone et al., 1984) are antagonistic, the habituation of the proboscis extension reflex is reduced in both mutants (Duerr and Quinn, 1982). In habituation of the landing response, both dunce and rutabaga mutants habituate more rapidly (Rees and Spatz, 1989). In another paradigm, the giant fiber-mediated escape response, habituation is reduced in rutabaga mutants and slightly accelerated in dunce mutants (Engel and Wu, 1996). At first glance, these contradictory effects of the dunce and rutabaga mutants on different behavioral paradigms is not surprising, because rutabaga and dunce gene products are expressed differently within the brain (Nighorn et al., 1991; Han et al., 1992), and the contribution of particular neuronal circuits to different behaviors is not identical. In the case of the rutabaga, it has been demonstrated recently that only a small neural circuit accounts for the defects of the rutabaga mutant in distinct aspects of olfactory learning. The rescue of rutabaga adenylyl cyclase activity in a subset of Kenyon cells is sufficient to rescue the behavioral defect of rutabaga in olfactory short-term memory (Zars et al., 2000). Similar rescue experiments can provide substantial information about which brain areas contribute to habituation as tested by the different paradigms in Drosophila.

\section{Distinct mechanisms contribute to habituation and dishabituation}

In agreement with the findings from various habituation paradigms in Drosophila, the cAMP/PKA pathway is implicated in the habituation of the proboscis extension reflex in honeybees. In all of these cases, the $\mathrm{cAMP} / \mathrm{PKA}$ system seems to contribute to habituation regardless of the sensory modality used for the stimulation. In the honeybee, however, the cAMP/PKA pathway seems to be required only for distinct aspects of habituation. Blocking of the cAMP/PKA pathway interferes with neither dishabituation nor spontaneous recovery from habituation in the honeybee. Such a contribution of the cAMP system to the dynamics of habituation but not to dishabituation has also been shown for the habituation of the giant fiber response in Drosophila (Engel and Wu, 1996). These findings suggest that habituation consists of different clearly separable processes. In the future, this clear distinction between the processes will allow for the identification of mechanisms underlying dishabituation and recovery from habituation.

Although a role of the cAMP/PKA cascade has been shown in various habituation paradigms using aversive as well as appetitive stimulation, it is still not clear whether the cAMP plays a conserved role in habituation regardless of the sensory modality. A detailed comparative analysis testing different habituation paradigms in a single species will be necessary to address this problem. It is clear, however, that signaling pathways other than the cAMP/PKA pathway contribute to habituation, as shown for the $\mathrm{Ca}^{2+} /$ calmodulin-dependent protein kinase (Jin et al., 1998).

\section{NO/cGMP-mediated PKA activation in antennal lobes contributes to habituation}

Although in Drosophila the site of action of the cAMP pathway is unknown, we identified the $\mathrm{CAMP} / \mathrm{PKA}$ pathway in antennal lobes as the site that contributes to this distinct component of habituation. The detailed analysis of the modulation of the PKA during habituation uncovered two pharmacologically dissectible components (Fig. 2): a fast, transient PKA activation that requires monoamines (Hildebrandt and Müller, 1995a,b) and a slow component of PKA activation that is highly correlated with response probability during habituation (Figs. 3, 4). The latter is mediated by the NO/cGMP system within each AL circuitry (Fig. 6).

The distribution of the NO-producing enzyme, NO synthase (NOS), and PKA in the AL (Müller and Hildebrandt, 1995; Müller, 1997) point to the sensory neurons and the local interneurons of the AL as possible release sites of NO. Whereas the sensory neurons exhibit a weak PKA immunostaining and an intermediate degree of NOS labeling, the interneurons show very strong PKA and NOS staining. Because non-cell-permeable NO scavengers block the NO-mediated function during habituation (Fig. 5), the NO released from sensory neurons or interneurons most likely acts on neighboring target neurons that contain the soluble guanylate cyclase and the PKA. Although the details are yet unknown, the neural circuitry mediating signal integration during habituation seems to cover the entire AL. This notion is supported by the findings that (1) the slow component of PKA activation can be measured in each dissected fraction of a single AL and (2) uncaging of NO, cGMP, or cAMP in the entire AL imitates the behavioral effect.

A contribution of sensory neurons and interneurons to habituation has also been demonstrated in other organisms. In Drosophila, targeted expression of mutated enzymes has been used to address the function of mechanosensory neurons in habituation 
of a reflex controlling leg position (Jin et al., 1998). Manipulation of the $\mathrm{Ca}^{2+} /$ calmodulin-dependent protein kinase II, which is implicated in synaptic transmission, leads to a reduced reflex response and a severe disruption of habituation. This suggests that the mechanosensory neurons are critically involved in setting the threshold level of the postsynaptic circuit. However, the neural circuit postsynaptic to these mechanosensory neurons is presently unknown.

In C. elegans, lesions of identified neurons enabled the identification of the neural circuit that underlies the touch and tap withdrawal reflexes. These studies demonstrate that a few mechanosensory neurons, interneurons, and motoneurons contribute specifically to habituation (Chalfie et al., 1985; Wicks and Rankin, 1995; Rose and Rankin, 2001). A similar picture emerges from studies in Aplysia. Although homosynaptic depression is observed in mechanosensory neurons after repeated intracellular current injection, the plastic changes underlying habituation of the aversive tail and siphon withdrawal reflexes seem to occur in interneurons rather than in the sensory neurons (Stopfer and Carew 1996; Stopfer et al., 1996).

\section{Mechanisms of NO/cGMP-modulated PKA activation in the AL differ between habituation and associative learning}

Recently, we demonstrated that multiple-trial associative olfactory learning induces an NO/cGMP-mediated PKA activation in the AL that is involved in the induction of LTM (Müller, 2000). In both the induction of olfactory LTM and the appetitive reflex habituation, the NO/cGMP system specifically mediates PKA activation induced by repeated stimulation. A comparison, however, reveals strong evidence that different cellular networks and mechanisms contribute to the function of the NO/cGMP-system in habituation and induction of associative olfactory LTM.

In contrast to the stimulation with one sensory modality in habituation, a distinct temporal pairing of stimuli from two sensory modalities is required to induce NO/cGMP-mediated PKA activation in associative learning. Only the repeated forward pairing of an odor stimulus [conditioned stimulus (CS)] with a sucrose stimulus [unconditioned stimulus (US)] leads to $\mathrm{NO} /$ cGMP-mediated PKA activation in associative learning. The latter occurs in both ALs, whereas during habituation, NO/ cGMP-mediated PKA activation is restricted to one AL. This finding points to basic differences in the induction mechanisms.

The temporal dynamics of PKA activation induced by either habituation or associative learning also differ from each other. Whereas in habituation the contribution of the NO/cGMP system is detectable after 10 repeated sucrose stimulations with an ISI of $1 \mathrm{sec}$, the NO/cGMP-mediated PKA activation in associative learning is evident after 3 CS-US pairings with an ISI in the range of minutes.

Because of the fact that NO synthesis is $\mathrm{Ca}^{2+}$ dependent, different cellular scenarios have to be postulated with respect to the release sites of NO in habituation and associative learning. Repeated sucrose stimulus during habituation induces NO release from yet unknown but presumably constant and defined release sites within the AL. In associative olfactory learning, the pattern of NO release is most likely restricted to a characteristic subset of glomeruli activated by the particular odor (Joerges et al., 1997). In both cases, NO acts via regulation of cGMP levels in the target cells. However, our investigations show that the mechanisms of the NO-mediated interactions differ between habituation and associative learning. Whereas local uncaging of NO in the entire AL imitates the presumed behavioral effect in habituation, uncaging NO in the ALs not only fails to imitate the presumed effect on induction of long-term memory but impairs learning in general (Müller, 2000). This supports the idea that uncaging NO in the entire AL interferes with yet unknown NO-mediated processes required for olfactory learning. These processes are apparently not modulated via cGMP and PKA, because uncaging cGMP and cAMP in the entire AL does not impair olfactory learning and improves LTM formation as predicted.

Together, all of these observations point to different NOmediated processes for habituation and associative learning. A characteristic these processes have in common, however, is that the NO system in the ALs plays an important role in translating temporal features of stimulation into distinct temporal activation patterns of intracellular pathways such as the cAMP/PKA pathway.

\section{REFERENCES}

Abel T, Nguyen PV, Barad M, Deuel TAS, Kandel ER (1997) Genetic demonstration of a role for PKA in the late phase of LTP and in hippocampus-based long-term memory. Cell 88:615-626.

Asztalos Z, Von Wegerer J, Wustmann G, Dombradi V, Gausz J, Spatz H-C, Friedrich P (1993) Protein phosphatase 1-deficient mutant Drosophila is affected in habituation and associative learning. J Neurosci 13:924-930.

Bailey CH, Chen M (1983) Morphological basis of long-term habituation and sensitization in Aplysia. Science 220:91-93.

Bailey CH, Chen M (1988) Morphological basis of short-term habituation in Aplysia. J Neurosci 8:2452-2459.

Boyle MB, Klein M, Smith SJ, Kandel ER (1982) Serotonin increased intracellular $\mathrm{Ca}^{2+}$ transients in voltage-clamped sensory neurons of Aplysia californica. Proc Natl Acad Sci USA 81:7642-7646.

Braun G, Bicker G (1992) Habituation of an appetitive reflex in the honeybee. J Neurophysiol 67:588-598.

Byers D, Davis RL, Kiger JAJ (1981) Defect in cyclic AMP phosphodiesterase due to the dunce mutation of learning in Drosophila melanogaster. Nature 289:79-81.

Byrne JH (1982) Analysis of synaptic depression contributing to habituation of gill-withdrawal reflex in Aplysia californica. J Neurophysiol 48:431-437

Carew TJ, Pinsker H, Kandel ER (1972) Long-term habituation of a defensive withdrawal reflex in Aplysia. Science 175:451-454.

Castellucci VF, Pinsker H, Kupfermann I, Kandel ER (1970) Neuronal mechanisms of habituation and dishabituation of the gill-withdrawal reflex in Aplysia. Science 167:1745-1748.

Chalfie M, Sulston JE, White JG, Southgate E, Thomson JN, Brenner S (1985) The neural circuit for touch sensitivity in Caenorhabditis elegans. J Neurosci 5:956-964.

Davis RL (1996) Physiology and biochemistry of Drosophila learning mutants. Physiol Rev 76:299-317.

Duerr JS, Quinn WG (1982) Three Drosophila mutations that block associative learning also affect habituation and sensitization. Proc Natl Acad Sci USA 79:3646-3650.

Engel JE, Wu CF (1996) Altered habituation of an identified escape circuit in Drosophila memory mutants. J Neurosci 16:3486-3499.

Engel JE, Wu CF (1998) Genetic dissection of functional contributions of specific potassium channel subunits in habituation of an escape circuit in Drosophila. J Neurosci 18:2254-2267.

Groves PM, Thompson RF (1970) Habituation: a dual process theory. Psychol Rev 77:419-450.

Han P-L, Levin LR, Reed RR, Davis RL (1992) Preferential expression of the Drosophila rutabaga gene in mushroom bodies, neural centers for learning in insects. Neuron 9:619-627.

Hildebrandt H, Müller U (1995a) Octopamine mediates rapid stimulation of protein kinase $\mathrm{A}$ in the antennal lobe of honeybees. J Neurobiol 27:44-50.

Hildebrandt H, Müller U (1995b) PKA activity in the antennal lobe of honeybees is regulated by chemosensory stimulation in vivo. Brain Res 679:281-288.

Jin R, Griffith LC, Murphey RK (1998) Presynaptic calcium/calmodulindependent protein kinase II regulates habituation of a simple reflex in adult Drosophila. J Neurosci 18:8955-8964.

Joerges J, Küttner A, Galizia G, Menzel R (1997) Representations of odours and odour mixtures visualized in the honeybee brain. Nature 387:285-288.

Krasne FB (1969) Excitation and habituation of the crayfish escape reflex: the depolarizing response in lateral fibres of the isolated abdomen. J Exp Biol 50:117-157. 
Livingstone MS, Sziber PP, Quinn WG (1984) Loss of calcium/calmodulin responsiveness in adenylate cyclase of rutabaga, a Drosophila learning mutant. Cell 37:205-215.

Müller U (1997) Neuronal cAMP-dependent protein kinase type II is concentrated in Mushroom bodies of Drosophila melanogaster and the honeybee, Apis mellifera. J Neurobiol 33:33-44.

Müller U (2000) Prolonged activation of cAMP-dependent protein kinase during conditioning induces long-term memory in honeybees. Neuron 27:159-168.

Müller U, Hildebrandt H (1995) The nitric oxide/cGMP system in the antennal lobe of Apis mellifera is implicated in integrative processing of chemosensory stimuli. Eur J Neurosci 7:2240-2248.

Nighorn A, Healy MJ, Davis RL (1991) The cyclic AMP phosphodiesterase encoded by the Drosophila dunce gene is concentrated in the mushroom body neuropil. Neuron 6:455-467.

Rankin CH (1994) Mechanistic questions raised by a behavioral analysis of habituation in Caenorhabditis elegans. The Neuroscientist 6:3-9.

Rayport S, Schacher S (1986) Synaptic plasticity in vitro cell culture of identified Aplysia neurons mediating short term-habituation and sensitization. J Neurosci 6:759-763.

Rees CT, Spatz H-C (1989) Habituation of the landing response of
Drosophila wild-type and mutants defective in olfactory learning. J Neurogenet 5:105-118.

Rose JK, Rankin CH (2001) Analyses of habituation in Caenorhabditis elegans. Learn Mem 8:63-69.

Schacher S, Castellucci VF, Kandel ER (1988) cAMP evokes long-term facilitation in Aplysia sensory neurons that requires new protein synthesis. Science 240:1667-1669.

Stopfer M, Carew TJ (1996) Heterosynaptic facilitation of tail sensory neuron synaptic transmission during habituation in tail-induced tail and siphon withdrawal reflexes of Aplysia. J Neurosci 16:4933-4948.

Stopfer M, Chen XH, Tai YT, Huang GS, Carew TJ (1996) Site specificity of short-term and long-term habituation in the tail-elicited siphon withdrawal reflex of Aplysia. J Neurosci 16:4923-4932.

Thompson RF, Spencer WA (1966) Habituation: a model phenomenon for the study of the neuronal substrates of behavior. Psychol Rev 73:16-43.

Wicks SR, Rankin CH (1995) Integration of mechanosensory stimuli in Caenorhabditis elegans. J Neurosci 15:2434-2444.

Zars T, Fischer M, Schulz R, Heisenberg M (2000) Localization of a short-term memory in Drosophila. Science 288:672-675. 